\title{
ERRORES DE REDONDEO EN UN COMPUTADOR
}

JURGEN WEYER

A. NUMEROS DE UN COMPUTADOR.

Cada computador puede representar sólo números con una mantisa de $k$ cifras, ( $k$ depende del modelo). Por eso los números de la máquina tienen la forma:

$$
z= \pm \cdot d_{1} d_{2} \ldots d_{k} \cdot 10^{\ell}
$$

donde las cifras de la mantisa son $d_{i} \varepsilon\{0,1, \ldots 9\} ; \ell$ es el exponente. La representación siempre es normalizada,ó sea $d_{1} \neq 0$ (Internamente el computador utiliza la base 2 en lugar de 10).

Si se quiere computarizar un número real arbitra $r$ io $x$, se tiene que aproximar este número por un número de la máquina. Las operaciones para aproximarlo son las siguien tes :
a) Normalizar $x$
b) Redondear $x$ a $k$ cifras de la mantisa
El resultado de estas operaciones es : 


$$
\begin{gathered}
\bar{x}=r d(x) \\
\Delta x=r d(x)-x \text { es el error absoluto del input } \\
\varepsilon_{x}=\Delta x / x \quad \text { es el error relativo del input } \\
\text { Además : } \\
\bar{x}=x\left(1+\varepsilon_{x}\right) \\
\text { eps }=10^{-k} \mid \leqslant \text { eps }
\end{gathered}
$$

se llama exactitud del computador.

B. OPERACIONES ELEMENTALES.

Para números $a, b$ de la máquina las operaciones elementales $+,-, \cdot, /$, son calculados por el proceso siguiente :

a) a,b son normalizados.

b) El resultado $z=a+b, a-b, a . b, a / b$, es calculado con $2_{k}$ cifras.

c) $z$ es normalizado.

d) $r d(z)$ es calculado.

rations :

Estas operaciones se llaman floating-point-ope

$$
f \ell(a+b), f \ell(a-b), f l(a ; b), f l(a / b)
$$

butivas.

Estas operaciones no son asociativas ni distri

ce :

El error relativo de estas operaciones satisfa

$$
\left.\begin{array}{l}
f \ell(a \pm b)=(a \pm b)(1+\varepsilon) \\
f \ell(a \cdot b)=a \cdot b \quad(1+\varepsilon) \\
f \ell(a / b)=a / b \quad(1+\varepsilon)
\end{array}\right\} \mid \begin{array}{ll}
\mid c p s
\end{array}
$$

e. PROPAGACION DE UN ERROR EN UN ALGORITMO.

Definamos un algoritmo como una secuencia de 
operaciones elementales que prescribe como se puede calcular la solución de un problema, utilizando los datos del input.

Estudiamos como los datos alterados del input $v$ las alteraciones en el calculo influyen el resultado.

\section{INPUT EXACTO, SOLO ALTERACIONES EN EL CALCULO.}

Ejemplo : Producto interior

$s=\sum_{i=1}^{n} a_{i} b_{i}$, donde $a_{i}, b_{i}$ son números de la mâquina

\section{ALGORITMO}

$t_{1}:=a_{1} \cdot b_{1}, s_{1}:=t_{1}$

para $r=2, \ldots \ldots, n$

$t_{r}:=a_{r} \cdot b_{r}, s_{r}: s_{r-1}+t_{r}$

$s=s_{n}$

Realización con floating-point-operation :

$$
\begin{aligned}
s_{1}^{\prime}=t_{1}^{\prime} & =f l\left(a_{1} \cdot b_{1}\right)=a_{1} \cdot b_{1}\left(1+\mu_{1}\right) \\
t_{r}^{\prime} & =f \ell\left(a_{r} \cdot b_{r}\right)-a_{r} \cdot b_{r}\left(1+\mu_{r}\right) \\
s_{r}^{\prime} & =f l\left(s_{r-1}^{\prime}+t_{r}^{\prime}\right)=\left\{s_{r-1}^{\prime}+a_{r} b_{r}\left(1+\mu_{r}\right)\right\} \cdot\left(1+a_{r}\right)
\end{aligned}
$$

Donde $\left|\alpha_{r}\right|,\left|\mu_{r}\right| \leqslant$ eps

(*) $f l\left(\sum_{i=1}^{n} \quad a_{i} b_{i}\right)=s^{\prime}=\sum_{i=1}^{n} a_{i} b_{i}\left(1+\varepsilon_{i}\right)$, donde 


$$
\begin{aligned}
& \left(1+\varepsilon_{1}\right)=\left(1+\alpha_{1}\right)\left(1+\alpha_{2}\right)\left(1+\alpha_{3}\right) \ldots \ldots\left(1+\alpha_{n}\right) \\
& \left(1+\varepsilon_{r}\right)=\left(1+\mu_{r}\right)\left(1+\alpha_{r}\right)\left(1+\alpha_{r+1}\right) \ldots\left(1+\alpha_{n}\right), r=2, \ldots n \\
& \left|\left(1+\varepsilon_{r}\right)\right| \leqslant(1+\text { eps })^{n}=1+n \text {. eps }
\end{aligned}
$$

El error relativo en el resultado es menor que $n$. eps. Interpretación de (*) : Hemos calculado exactamente el producto escalar para un input, que es un poco alterado.

Datos del input :

$$
\left(\begin{array}{l}
a_{1} \\
\dot{b} \\
a_{n}
\end{array}\right)\left(\begin{array}{cc}
b_{1} & \left(1+\varepsilon_{1}\right) \\
\cdot & \\
\cdot \\
b_{n} & \left(1+\varepsilon_{n}\right)
\end{array}\right)
$$

El resultado es aceptable.

2. INPUT ALTERADO, CALCULO EXACTO.

$$
\begin{aligned}
& x=\left(\begin{array}{c}
x_{1} \\
\cdot \\
\cdot \\
x_{n}
\end{array}\right) \text { sean los daros exactos del input } \\
& y=\left(\begin{array}{l}
y_{1} \\
\cdot \\
\cdot \\
\cdot \\
y_{n}
\end{array}\right) \varepsilon i^{n}, \text { sean los resultados deseados }
\end{aligned}
$$


$\psi$ sea un instrucción para calcular y.

Entonces : $\psi$ es una aplicación

$$
\psi: R^{n} \longrightarrow R^{m}, \psi \varepsilon c^{k}, \psi(x)=y
$$

$\bar{x}$ sea un aproximación de $x$ (error en el input).

Error absoluto: $\Delta \mathrm{x}_{i}=\overline{\mathrm{x}}_{i}-\mathrm{x}_{i}, \Delta \mathrm{x}=\overline{\mathrm{x}}-\mathrm{x}$ Error relativo $: \varepsilon_{x i}=\Delta x_{i} / x_{i}$

$$
y=\psi(x), \bar{y}=\psi(\bar{x})
$$

Error en el resultado:

$$
\Delta y_{i}=\bar{y}_{i}-y_{i}=\psi_{i}(\bar{x})-\psi_{i}(x)=
$$

$=\Sigma \frac{\partial_{i}}{\partial x_{j}} \Delta x_{j} \frac{1}{2} \sum_{p, q=1}^{n} \frac{\partial^{2}}{\partial x_{p} \partial x_{q}} \Delta x_{p} \Delta x_{q}+\cdots$

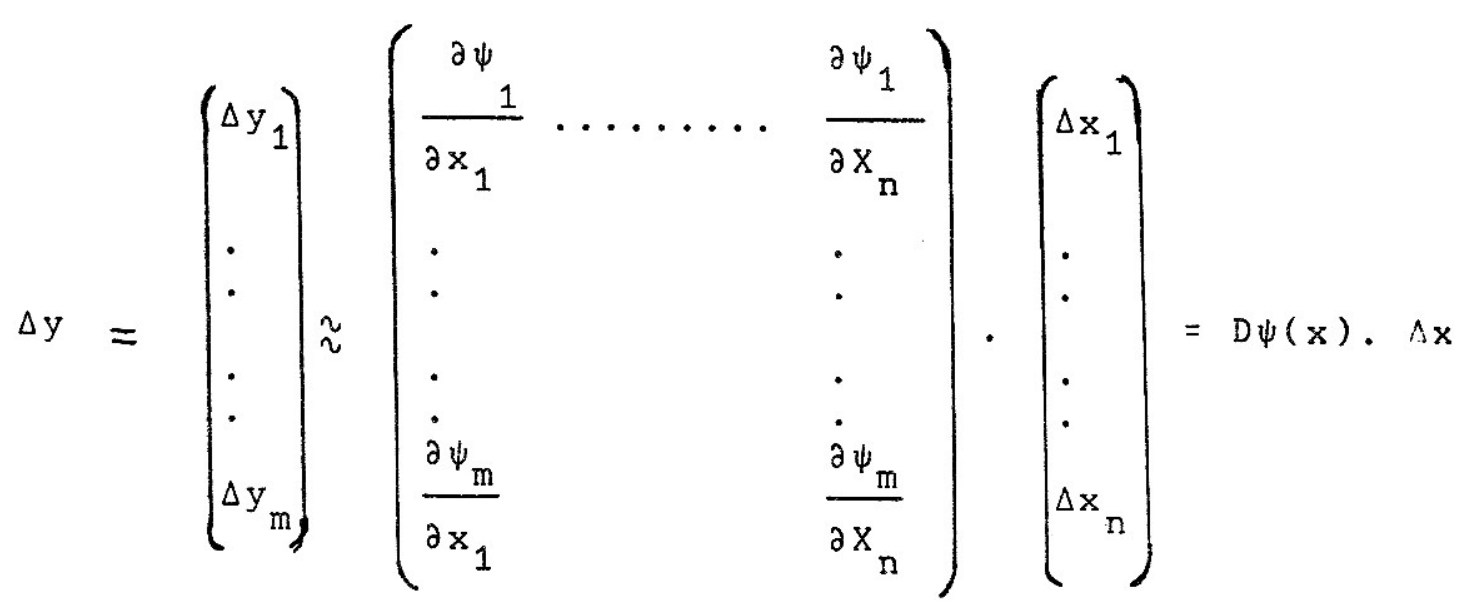
un input alterado.

$\Delta y$ es el error en el resultado en consecuencia de 


$$
\varepsilon_{y_{i}}=\sum_{j=1}^{n} \frac{x_{j}}{\psi(x)} \frac{\partial \psi_{i}(x)}{\partial x_{j}} \varepsilon_{x_{j}}
$$

es el error relativo. Los números $\frac{x_{j}}{\psi_{i}} \frac{\partial \psi_{i}}{\partial x_{j}}$

se llaman números de la condición. Cuando estos números son $>>1(<1)$, el error en el input tiene gran (poca) importancia para el resultado.

\section{EJEMPLOS :}

$$
\begin{aligned}
& \psi\left(x_{1}, x_{2}\right)=x_{1}, x_{2} \rightarrow \varepsilon_{\psi}=\varepsilon_{x_{1}}+\varepsilon_{x_{2}} \\
& \psi\left(x_{1}, x_{2}\right)=x_{1} / x_{2} \rightarrow \varepsilon_{\psi}=x_{1}-x_{2}
\end{aligned}
$$

$\psi\left(x_{1}, x_{2}\right)=x_{1} \pm x_{2} \quad \varepsilon_{\psi}=\frac{x_{1}}{x_{1} \pm x_{2}} \varepsilon_{x_{1}} \pm \frac{x_{2}}{x_{1} \pm x_{2}} \varepsilon_{2}$

$$
\psi(x)=\sqrt{x} \rightarrow \varepsilon_{\psi}=\frac{1}{2} \varepsilon_{x}
$$

3. INPUT ALTERADO, ALTERACIONES EN EL CALCULO.

ANALISIS DIFERENCIAL PARA PROPAGACION DE ERRORES.

Sea $\psi: R^{n} \rightarrow R^{m}$ la aplicación, que descri be nuestro problema del cálculo $\psi(x)=y$. Cada algoritmo está representado por una secuencia de operaciones elementales $\psi^{(i)}$.

Por eso descomponemos wen: 
$\psi=\psi^{(r)} \circ \psi^{(r-1)} \circ \ldots \ldots \psi^{(1)} \circ \ldots \ldots \psi^{(0)}$

\section{EJEMPLO :}

$\psi(a, b)=a^{2}-b^{2}=(a+b)(a-b)$

Algoritmo 1: $\psi^{(0)}(a, b)=\left(\begin{array}{l}a^{2} \\ b^{2}\end{array}\right) \cdot \psi^{(1)}(a, b)=a-1$

entonces

$\psi=\psi^{(1)} \circ \psi^{(0)}$

$\begin{aligned} \text { Algoritmo } 2: \quad \bar{\psi}^{(0)}(a, b) & =\left(\begin{array}{l}a+b \\ a-b\end{array}\right), \bar{\psi}^{(1)}(a, b)=a \cdot b \\ \text { entonces } & \psi=\bar{\psi}\end{aligned}$

Se quiere elegir un algoritmo tal que el error en el resultado tenga poca importancia. Por eso se define las aplicaciones :

$$
\phi^{(0)}=\psi \quad \phi^{(i)}=\psi^{(r)} \circ \ldots \circ \psi^{(i)}
$$

Estas funciones ${ }^{(i)}$ se llaman aplicaciones rema nentes. Al calcular el resultado provisional

$\psi^{(i)}\left(x^{(i)}\right)$ el computador, calcula $\psi^{(i)}\left(x^{(i)}\right)+\alpha_{i+1}$ en lu․ gar de $\psi^{(i)}\left(x^{(i)} ; \alpha_{i+1}\right.$ es el error absoluto al aplicar $\psi^{(i)}$. Ahora se puede interpretar $\alpha_{i+1}$ como el error del input para la aplicación remanente $\phi^{(i+1}$ La influencia de este error $\alpha_{i+1}$ para el resultado final es 
D $\psi^{(i+1)} \cdot \alpha_{i+1}$ para el resultado final es $D^{(1+1)} \cdot \alpha_{i+1}$.

El error total en el resultado final es :

$$
\begin{aligned}
& \Delta y=D \phi^{(0)} a_{0}+D \phi^{(1)} \alpha_{1}+\ldots . .+\phi^{(r)} a_{r}+\alpha_{r+1} \\
& D \phi^{(0)} \alpha_{0}=D . \Delta x \text { es el error a consecuencia del input }
\end{aligned}
$$

falsificado.

$\sum_{i=1}^{r} D \psi^{(i)} \cdot \alpha_{i}+\alpha_{r+1}$ son los errores a consecuencia

del calculo. Para todos los algoritmos, con los que se puede calcular $\psi$, la influencia del error del input $D \psi$. $\Delta x$ es $10 \mathrm{mismo.} \mathrm{Un} \mathrm{algoritmo} \mathrm{se} \mathrm{llama} \mathrm{numéricamente} \mathrm{estable} \mathrm{si}$

D $\psi$. $\Delta x$ tiene el mismo orden de magnitud como $\sum_{i=1}^{r} D \phi^{(i)} \alpha_{i}+\alpha_{r+1}$. Para cada problema se tiene que

elegir entre los algoritmos posibles un algoritmo estable.

Ejemplo:

PROBLEMA : CaIcule $\cos (k \mathrm{x}) \mathrm{k} \varepsilon \mathrm{N}$

Dato del input: $\cos x$ (se calcula $\cos x$ solo en $\left[0, \frac{\pi}{2}\right]$ ) Algoritmo 1 :

recursión :

$$
\cos (i+1)=2 \cos x \cdot \cos i x-\cos (i-1) x \rightarrow
$$

$$
c_{i+1}=2 c_{1} c_{i}-c_{i-1}
$$

input : $\quad c_{0}=1, c_{1}=\cos x$,

$$
c_{i}=\cos i x
$$

Anâlisis del error

$\mathrm{x}+\psi_{1} c_{1}-\psi_{2} c_{2}-\ldots \ldots c_{i}-\ldots-c_{k}$ 


$$
c_{i}=\cos i x \quad x: \frac{1}{i} \operatorname{arc} \cos c_{i}
$$

$c_{k}=\phi^{(i)}\left(c_{i}\right)=\cos (k x)=\cos \left(\frac{k}{i} \operatorname{arcos} c_{i}\right) \Longrightarrow$

$D \phi^{(i)}(i)=+\operatorname{sen}\left(\frac{k}{i} \operatorname{arcos} c_{i}\right) \cdot \frac{k}{i} \frac{1}{\sqrt{1-c_{i}^{2}}}$

$$
=\frac{k}{i} \frac{\operatorname{sen} k x}{\operatorname{sen} i x} i>0
$$

$D \phi(0)=-k \operatorname{sen} k x$

$\Delta c_{k}=-k \operatorname{sen}(k x) \Delta x+\sum_{i=1}^{k} \frac{k \operatorname{sen} k x}{i \operatorname{sen} i x} \alpha_{i}+\alpha_{k+1}$

Análisis para $x \approx 0 \rightarrow \lim _{x \rightarrow 0} \frac{k \operatorname{sen} k x}{i \operatorname{sen} i x}=\frac{k^{2}}{i^{2}}$

$\rightarrow \quad$ la influencia del error $\alpha_{1}$ es $k^{2} \alpha_{1}$. Para $k=1000$ el factor del refuerzo del error es $10^{6}$. Por eso perdimos 6 cifras correctas en el resultado.

\section{Algoritmo 2 .}

$$
\begin{aligned}
d c_{i} & =\cos i x-\cos (i-1) x=2 \cos x \cdot \cos (i-1) x-\cos (i-1) x-\cos (i-2) x \\
& =2 \cos ((i-1) x)[\cos x-1]+d c_{i-1}
\end{aligned}
$$

recursión doble : $c_{i}=c_{i-1}+d c_{i}, d c_{i+1}=t c_{i}+d c_{i}$

input

$$
: c_{0}=1 ; \quad d c_{1}=\cos x-1=2 \operatorname{sen}^{2}\left(\frac{x}{2}\right) ; t=2 d C_{1}
$$

Analisis diferencial muestra : Algoritmo 2 es es table. Generalmente se puede decir: recursiones dobles son 
más estables o se prefiere iteraciones con 3 expresiones de la forma

$$
c_{i+1}=f\left(c_{i}, c_{i-1}\right) .
$$

\section{BIBLI OGRAF IA}

1.- Wilkinson; Rounding : Error in Algebraic Processes, her Majesty's Stationary office, London 1963.

2.- Wilkinson, Rudungsfehler, Heidelberger. Tashenbucher 44, springer 1969 .

3.- Wilkinso, Error Analysis of floating-point-computation Numer. Math 2. $319-340$.

4.- Stoer, Einfuhrung in die Numerische Mathematick I, Heidelberger Taschenbucher 105 Springer 1973. 